\title{
Meson transition form factors at KLOE/KLOE-2
}

\author{
Ivano Sarra ${ }^{1, a}$ for the KLOE-2 collaboration
}

${ }^{1}$ Laboratori Nazionali di Frascati - INFN -

\begin{abstract}
In this work we present a study of the Dalitz decay $\phi \rightarrow \eta \mathrm{e}^{+} \mathrm{e}^{-}$, with $\eta \rightarrow \pi^{0} \pi^{0} \pi^{0}$, performed using $1.7 \mathrm{fb}^{-1}$ of $\mathrm{e}^{+} \mathrm{e}^{-}$collisions collected at KLOE in the 2004-2005 data taking period. The resulting branching fraction is: $\operatorname{BR}\left(\phi \rightarrow \eta \mathrm{e}^{+} \mathrm{e}^{-}\right)=(1.059 \pm 0.007$ (stat. $) \pm 0.038$ (norm. $\left.)\right) \times 10^{-4}$. We have also studied the invariant mass of the dilepton system, extracting the slope of the transition form factor: $\mathrm{b}_{\phi \eta}=(1.17 \pm 0.10) \mathrm{GeV}^{-2}$. The $\phi \rightarrow \pi^{0} \mathrm{e}^{+} \mathrm{e}^{-}$rare decay is also being studied using the same data sample. The branching ratio will be measured, with a consistent reduction of the actual total uncertainty. The double off shell $\pi^{0}$ Transition Form Factor will also be measured for the first time in the time-like region, with the momentum transfer given by the $\mathrm{e}^{+} \mathrm{e}^{-}$invariant mass.
\end{abstract}

\section{Introduction}

Conversion decays of vector and pseudoscalar mesons $\left(\mathrm{A} \rightarrow \mathrm{B} \gamma^{*}\right)$ are closely related to corresponding radiative decays $(\mathrm{A} \rightarrow \mathrm{B} \gamma)$. The nature of the mesons and their underlying quark and gluon structure can be therefore described by the transition form factor function of $A$ into $B\left(F_{A B}\left(q^{2}\right)\right)$, that can be extracted from data by studying the probability of the conversion decay as a function of the squared invariant mass of the lepton pair.

The decay parametrization is taken from Ref. [1]:

$$
\begin{gathered}
\frac{d}{d q^{2}} \frac{\Gamma\left(\phi \rightarrow \eta e^{+} e^{-}\right)}{\Gamma(\phi \rightarrow \eta \gamma)}= \\
\frac{\alpha}{3 \pi} \frac{\left|F_{\phi \eta}\left(q^{2}\right)\right|^{2}}{q^{2}} \sqrt{1-\frac{4 m^{2}}{q^{2}}}\left(1+\frac{2 m^{2}}{q^{2}}\right)\left[\left(1+\frac{q^{2}}{m_{\phi}^{2}-m_{\eta}^{2}}\right)^{2}-\frac{4 m_{\phi}^{2} q^{2}}{\left(m_{\phi}^{2}-m_{\eta}^{2}\right)^{2}}\right]^{3 / 2}
\end{gathered}
$$

with

$$
F\left(q_{\phi \eta}^{2}\right)=\frac{1}{1-q^{2} / \Lambda_{\phi \eta}^{2}}
$$

in one-pole approximation. The form factor slope $b_{\phi \eta}=d F /\left.d q^{2}\right|_{q^{2}=0}$ is equal to $\Lambda_{\phi \eta}^{-2}$.

The naive vector meson dominance (VMD) approach is satisfactory in the description of some conversion decays, such as, for instance, the case of $\eta \rightarrow \gamma \mu^{+} \mu^{-}$, see Fig. 1(a), while failing for others, such as in the case of $\omega \rightarrow \pi^{0} \mu^{+} \mu^{-}$, see Fig. 1(b). Recently, new models have been developed (see

\footnotetext{
a e-mail: ivano.sarra@lnf.infn.it
} 
Ref. [2] and [3]) based on most accurate description of $\mathrm{A} \rightarrow \mathrm{B} \gamma^{*}$ channels. Therefore, it is important to validate this model with the experimental results. In this note, we focus our study on the decay channel $\phi \rightarrow \eta e^{+} e^{-}$, with $\eta \rightarrow 3 \pi^{0}$. The only existing data on $\phi \rightarrow \eta \mathrm{e}^{+} \mathrm{e}^{-}$come from the SND [4] and CMD-2 [5] experiments. Their $\operatorname{BR}\left(\phi \rightarrow \eta \mathrm{e}^{+} \mathrm{e}^{-}\right)$measurements are $(1.19 \pm 0.31) \times 10^{-4}$ and $(1.14 \pm 0.16) \times 10^{-4}$ respectively. The $\operatorname{VMD}$ expectation is $\operatorname{BR}\left(\phi \rightarrow \eta \mathrm{e}^{+} \mathrm{e}^{-}\right)=1.1 \times 10^{-4}$.

SND experiment has also measured the transition form factor from the $\mathrm{M}_{e e}$ invariant mass distribution on the basis of 213 events: $\mathrm{b}_{\phi \eta}=3.8 \pm 1.8 \mathrm{GeV}^{-2}$. The VMD expectation is $\mathrm{b}_{\phi \eta}=1 \mathrm{GeV}^{-2}$.
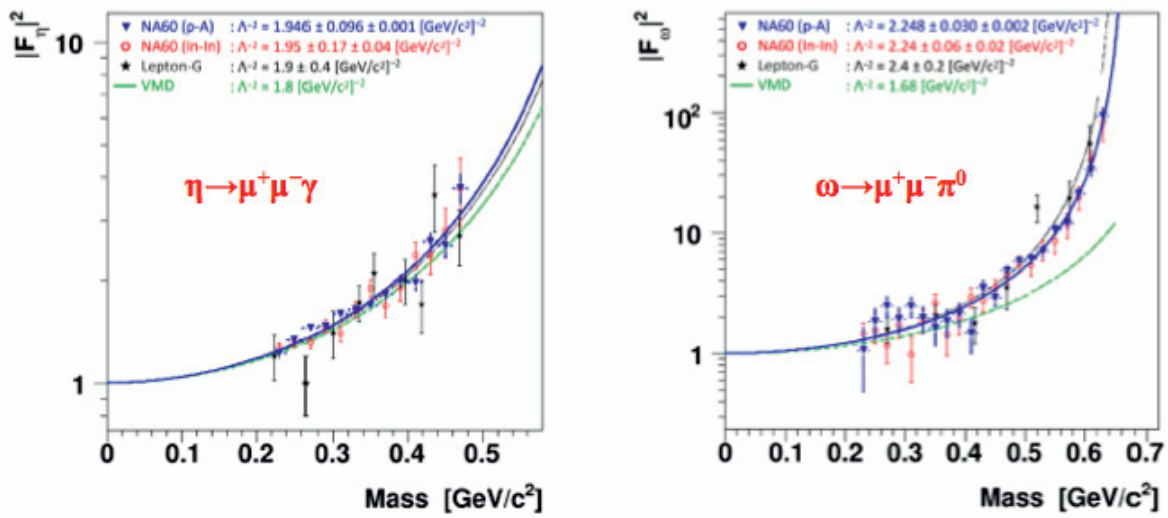

Figure 1. Form factor squared value as a function of the invariant mass of the lepton pair for the decay channels $\eta \rightarrow \gamma \mu^{+} \mu^{-}$(a) and $\omega \rightarrow \pi^{0} \mu^{+} \mu^{-}$(b) measured from Na60 experiment. In blue there are the experimental data and the fit line, in green VMD expectation, in black the Leupold's fit.

\section{The $\phi \rightarrow \eta e^{+} e^{-}$analysis}

A detailed study of $\phi \rightarrow \eta e^{+} e^{-}$decay is in progress, using $\eta \rightarrow \pi^{0} \pi^{0} \pi^{0}$ decay channel. The analyzed sample is based on $1.7 \mathrm{fb}^{-1}$ data, collected at $\sqrt{s}=m_{\phi}$ during the 2004-2005 KLOE data taking campaign.

The first step of the analysis is the rejection of splitted tracks using the broken-track algorithm [6], that retain only good track candidates coming from the interaction point.

The preselection requires:

1. one positive and one negative track in a cylinder around the interaction point (IP), with transverse radius $\mathrm{R}_{F V}=4 \mathrm{~cm}$ and total height $\mathrm{Z}_{F V}=20 \mathrm{~cm}$;

2. six photon candidates, i.e. energy deposits in the calorimeter without associated tracks with energy larger than $7 \mathrm{MeV}$, in an angular acceptance $\left|\cos \left(\theta_{\gamma}\right)\right|<0.92$ and in the expected time window for photon $\left(\left|\mathrm{T}_{\gamma}-\mathrm{R}_{\gamma} / \mathrm{c}\right|<\operatorname{MIN}\left(3 \sigma_{\mathrm{T}}, 2 \mathrm{~ns}\right)\right)$;

3. invariant mass of the six photons inside a window around the $\eta$ mass value, $400-700 \mathrm{MeV}$.

These preselection cuts allow to clearly identify the peak due to $\phi \rightarrow \eta e^{+} e^{-}$events in the distribution of the recoil mass to the $e^{+} e^{-}$pair, $M_{\text {recoil }}(e e)$ (see Fig. 2). 
The second peak at $\sim 590 \mathrm{MeV}$ is due to $\phi \rightarrow \mathrm{K}_{\mathrm{S}} \mathrm{K}_{\mathrm{L}}$, with $K_{S} \rightarrow \pi^{+} \pi^{-}$, having a wrong mass assignment. A $3 \sigma$ cut on the $M_{\text {recoil }}(e e)$ variable, $536.5<M_{\text {recoil }}(e e)<554.5 \mathrm{MeV}$, strongly reduces the background contribution.

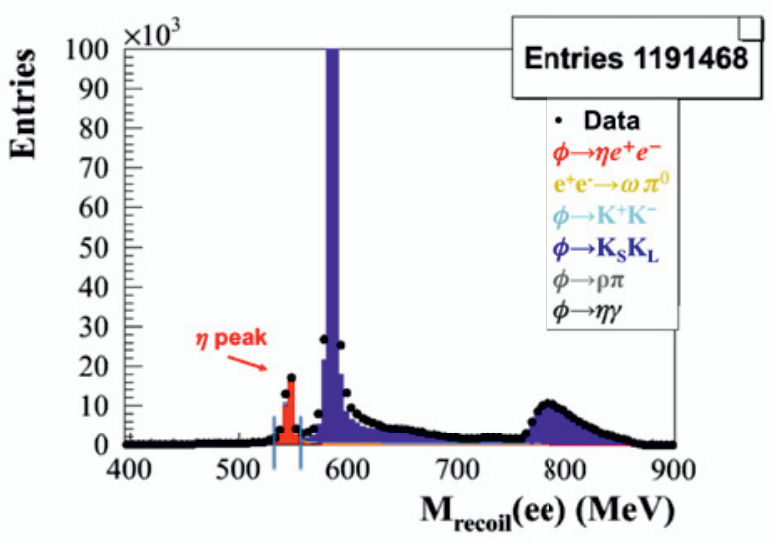

Figure 2. Recoil mass to the $e^{+} e^{-}$pair after the pre-selection cuts. Dots are data, single MC components are displayed in colors: cyan $\phi \rightarrow K^{+} K^{-}$, blue $\phi \rightarrow K_{S} K_{L}$, yellow $e^{+} e^{-} \rightarrow \omega \pi^{0}$, red $\phi \rightarrow \eta e^{+} e^{-}$, gray $\phi \rightarrow \rho \pi$, dark gray $\phi \rightarrow \eta \gamma$.

All MC distributions are normalized according to LSF production luminosity. The $\mathrm{M}_{e e}$ shape of the $\mathrm{MC}$ signal has been reshaped applying a hit-or-miss procedure, using the value of $1.2 \mathrm{GeV}^{-2}$.

Although a large part of the backgrounds is already rejected at this level, some contamination from photon conversion events, $\phi \rightarrow \eta \gamma$, with photon conversion on BP or DC walls, is still present. This background is rejected applying a specific photon-conversion recognition algorithm. The other background components, $\phi \rightarrow K_{S} K_{L}$, with $K_{S} \rightarrow \pi^{+} \pi^{-}$, and $e^{+} e^{-} \rightarrow \omega \pi^{0}$, are rejected using the time of flight to the calorimeter.

\subsection{Rejection of the photon conversion events}

Photons produced near the interaction region, can convert on the beam pipe (BP) or on drift chamber walls (DCW), simulating an $e^{+} e^{-}$pair from the interaction point. This residual background contamination, due mainly to $\phi \rightarrow \eta \gamma$ events, is rejected by tracking back to BP/DCW surfaces the two $e^{+}$, $e^{-}$candidates and then reconstructing the electron-positron invariant mass, $M_{e e}(B P / D C W)$, and the distance between the two particles, $\mathrm{D}_{\mathrm{ee}}(\mathrm{BP} / \mathrm{DCW})$. Both quantities are small if coming from photon conversion. In Fig. 3 the distributions for different samples are shown.

Photon conversions are removed by rejecting events with:

- $\mathrm{M}_{e e}(\mathrm{BP})<10 \mathrm{MeV}$ and $\mathrm{D}_{e e}(\mathrm{BP})<2 \mathrm{~cm}$,

- $\mathrm{M}_{e e}(\mathrm{DCW})<120 \mathrm{MeV}$ and $\mathrm{D}_{e e}(\mathrm{DCW})<4 \mathrm{~cm}$. 
EPJ Web of Conferences
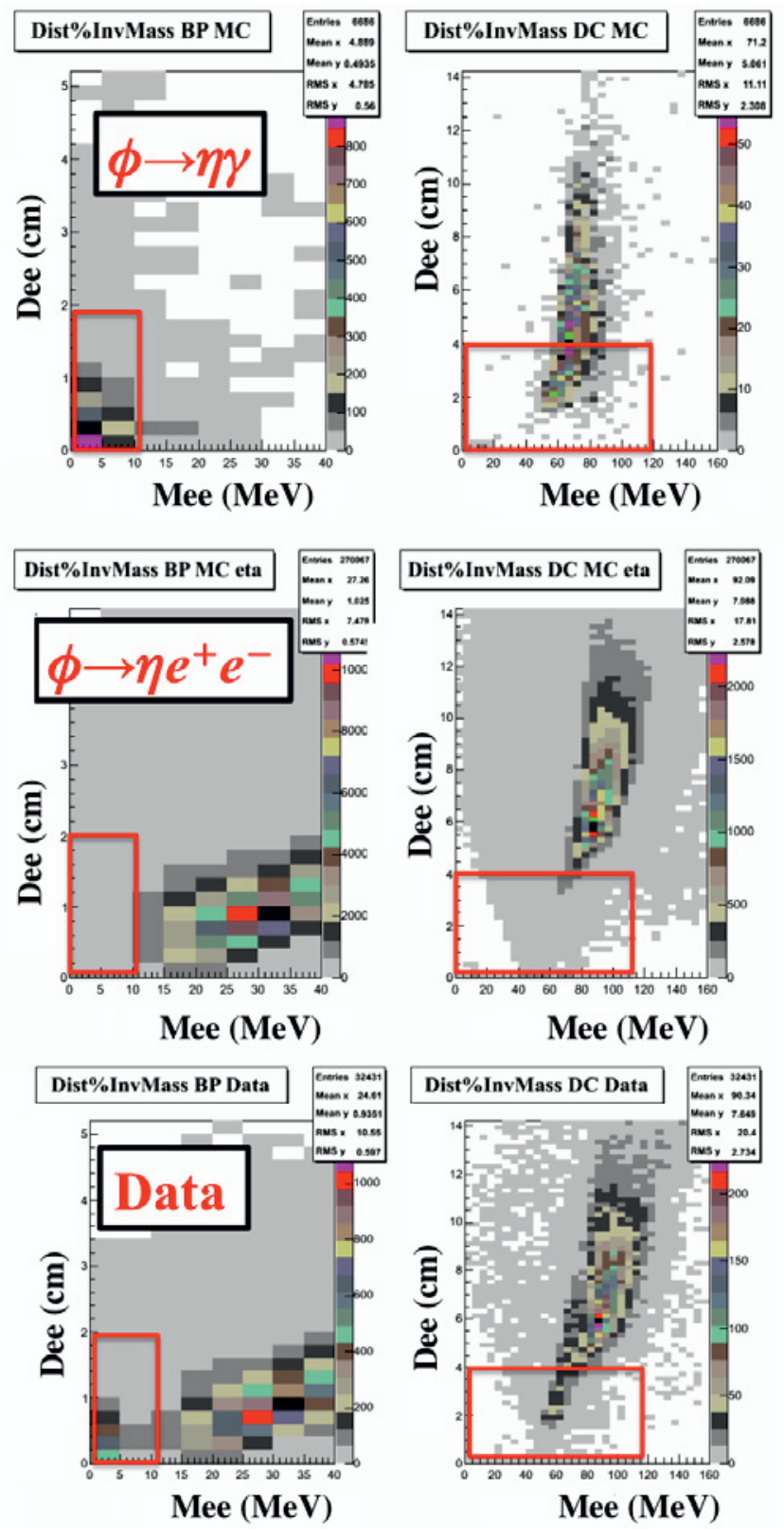

Figure 3. Conversion variables evaluated on the beam pipe (left panels) and on DC walls (right panels) after $M_{\text {recoil }}(e e)$ cut. Top: $\mathrm{MC} \phi \rightarrow \eta \gamma$, center: $\mathrm{MC} \phi \rightarrow \eta e^{+} e^{-}$, bottom: data. Events inside the red box are rejected. 


\subsection{Particle ID using the Time of Flight to the calorimeter}

The residual background contamination, originated from $\phi \rightarrow \mathrm{K}_{\mathrm{L}} \mathrm{K}_{\mathrm{S}}, \mathrm{K}_{\mathrm{S}} \rightarrow \pi^{+} \pi^{-}$and $\mathrm{K}_{\mathrm{L}} \rightarrow 3 \pi^{0}$, decays and $e^{+} e^{-} \rightarrow \omega \pi^{0}\left(\omega \rightarrow \pi^{+} \pi^{-} \pi^{0}\right)$ events, has two charged pions in the final state and is suppressed using the time of flight (TOF) of tracks to the calorimeter. When an EMC cluster is connected to a track, the arrival time to the calorimeter is evaluated both using the calorimeter timing $\left(T_{\text {cluster }}\right)$ and the track trajectory $\left(T_{\text {track }}=L_{\text {track }} / \beta c\right)$. The $D T=T_{\text {track }}-T_{\text {cluster variable is then evaluated for the }}$ electron $\left(D T_{e}\right)$ hypothesis. Events with an $e^{+}$OR $e^{-}$candidate inside a $3 \sigma$ 's window on the $D T_{e}$ variables $\left(-0.9<D T_{e}<0.62\right)$ are kept for the analysis. Since almost $100 \%$ of the events have at least an $e^{+}$or an $e^{-}$connected to a calorimeter cluster, the cut is very efficient on signal events. In Figure 4 the data-MC comparison for the $D T_{e}$ variable before (left) and after (right) the TOF cut is shown.
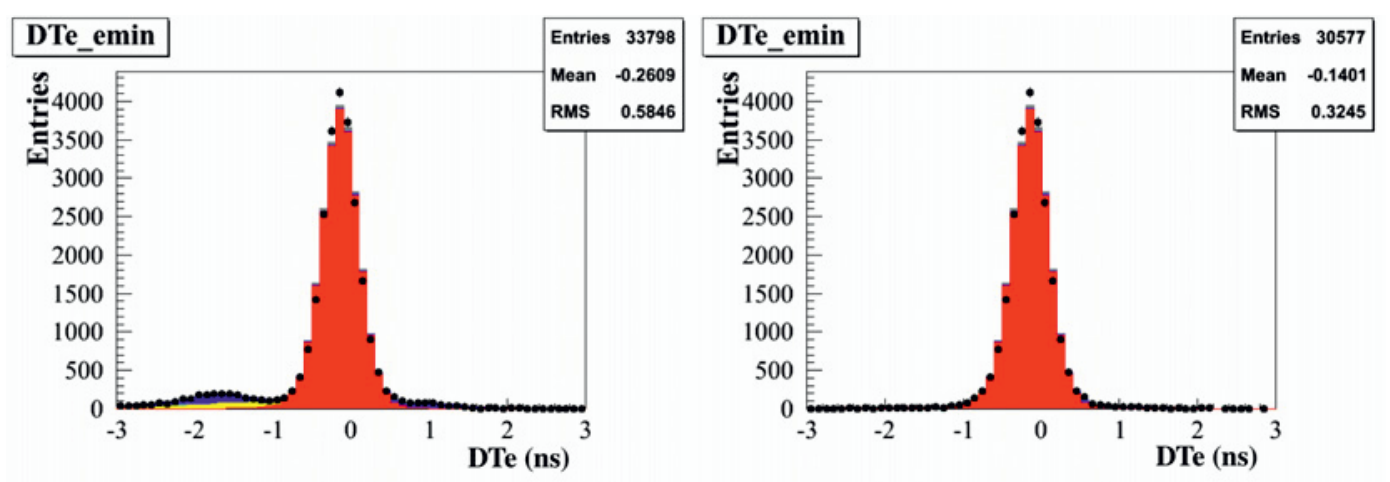

Figure 4. Data/MC comparison of arrival time to the calorimeter using the calorimeter timing $\left(T_{\text {cluster }}\right)$ and the track trajectory $\left(T_{\text {track }}=L_{\text {track }} / \beta c\right)$ in the electron hypothesis. The $D T_{e}=T_{\text {track }}-T_{\text {cluster }}$ variable before (left) and after (right) the TOF cut is showed. Dots are data, single MC components are displayed in colors: cyan $\phi \rightarrow K^{+} K^{-}$, blue $\phi \rightarrow K_{S} K_{L}$, yellow $e^{+} e^{-} \rightarrow \omega \pi^{0}$, red $\phi \rightarrow \eta e^{+} e^{-}$, gray $\phi \rightarrow \rho \pi$, dark gray $\phi \rightarrow \eta \gamma$.

In Figures 5, 6 left, data-MC comparison for the invariant mass, the polar angle of the $e^{+} e^{-}$pair and the $\cos \psi^{*}$ variable (the angle between the $\eta$ and the $e^{+}$in the $e^{+} e^{-}$rest frame), after the TOF selection, are shown.

The background is reduced by a factor of five as shown in Figure 6 right. 
EPJ Web of Conferences
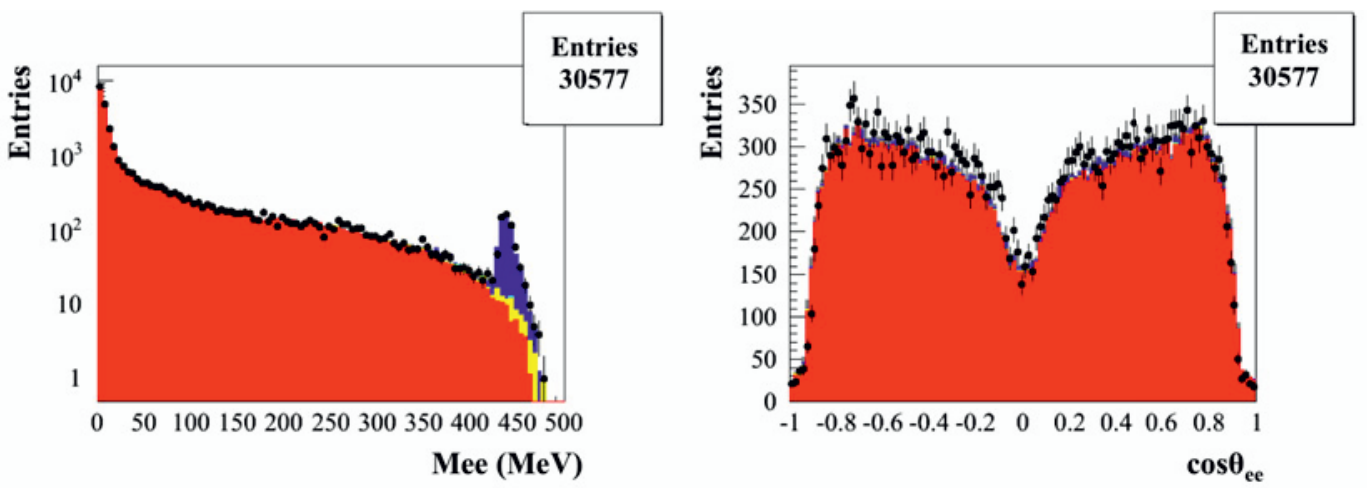

Figure 5. Data/MC comparison for the invariant mass (left) and the polar angle (right) of the $e^{+} e^{-}$pair after the time of flight cut. Dots are data, single MC components are displayed in colors: cyan $\phi \rightarrow K^{+} K^{-}$, blue $\phi \rightarrow K_{S} K_{L}$, yellow $e^{+} e^{-} \rightarrow \omega \pi^{0}$, red $\phi \rightarrow \eta e^{+} e^{-}$, gray $\phi \rightarrow \rho \pi$, dark gray $\phi \rightarrow \eta \gamma$.

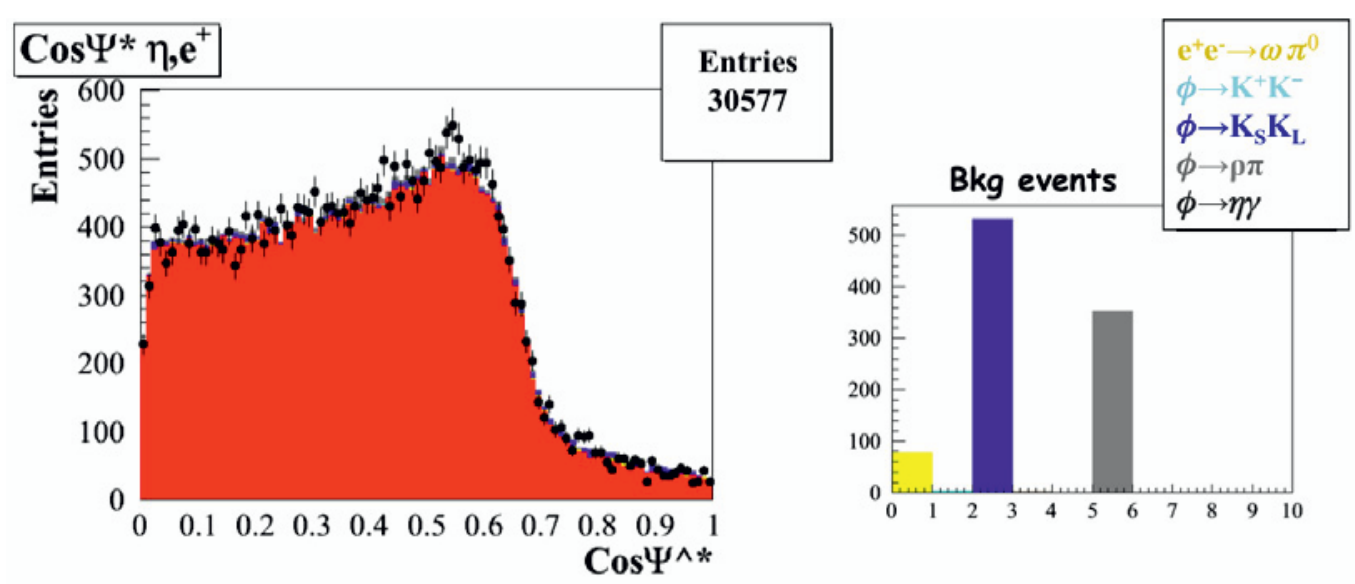

Figure 6. Data/MC comparison for the $\cos \psi^{*}$ variable (left) and summary of the background contamination (right) after the time of flight cut. The abscissa corresponds to the $\phi$ decay identification code of the GEANTFI package. 
At the end of the analysis chain, the residual background contamination is at the level of $3 \%$. After the background subtraction $29625 \pm 178 \phi \rightarrow \eta \mathrm{e}^{+} \mathrm{e}^{-}, \eta \rightarrow 3 \pi^{0}$, candidates remain in the analysis dataset.

In Fig. 7, the comparison between data and Monte Carlo events for the $M_{e e}$ distribution after the background subtraction is shown.
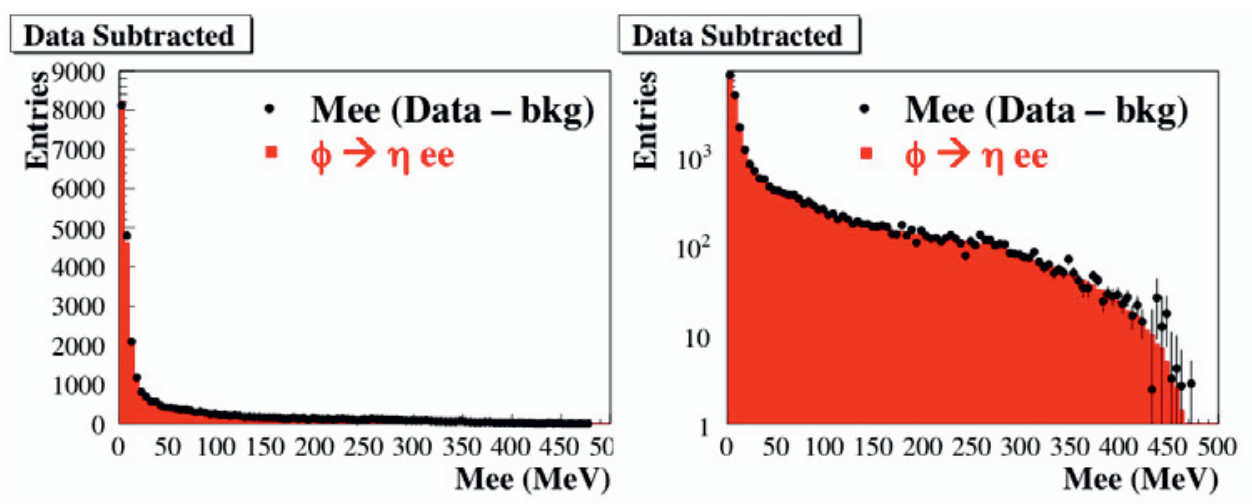

Figure 7. $\mathrm{M}_{e e}$ spectrum after all analysis cuts and background subtraction, compared with MC distribution for signal events, in linear and logarithmic scale.

\subsection{Branching ratio of the $\phi \rightarrow \eta e^{+} e^{-}$decay}

The total value for the efficiency, defined as the ratio between the number of generated events and the number of the events at the end of the analysis path, is $15.45 \%$ with a negligible error. Thus, we can measure the branching ratio of the $\phi \rightarrow \eta e^{+} e^{-}$decay using the formula:

$$
N_{\text {signal }}=\sigma_{e^{+} e^{-} \rightarrow \phi} \times \mathcal{L} \times B R\left(\phi \rightarrow \eta e^{+} e^{-}\right) \times B R\left(\eta \rightarrow 3 \pi^{0}\right) \times \epsilon
$$

The luminosity measurement is obtained using large angle Bhabha scattering events [7], giving $\mathcal{L}=1.68 \pm 0.01 \mathrm{fb}^{-1}$. We used the effective $\phi$ production cross section which takes into account the center of mass energy variations (1\%), [8]: $\sigma=3310 \pm 120 \mathrm{nb}$. The value of the $\operatorname{BR}\left(\eta \rightarrow 3 \pi^{0}\right)$ is taken from [9]: $\mathrm{BR}=(32.57 \pm 0.23 \%)$. The resulting value for the $\mathrm{BR}$ is:

$$
B R\left(\phi \rightarrow \eta e^{+} e^{-}\right)=(1.059 \pm 0.007(\text { stat } .) \pm 0.038(\text { norm } .)) \times 10^{-4}
$$

where the last term includes uncertainties on luminosity and $\phi$ production cross section.

This value is in agreement with the VMD predictions and consistent with the measurements from SND and CMD-2 experiments, however the accuracy is improved by a factor ten and five, respectively. 


\subsection{Fit to the Mee spectrum and extraction of the Form Factor Slope}

The fit procedure is performed minimising the $\chi^{2}$ function and it is based on the MINUIT package [10], is applied to the raw $\mathrm{M}_{e e}$ distribution, after applying a bin by bin background subtraction. The binning width used is $5 \mathrm{MeV}$. Analysis efficiency and smearing effects have been folded into the theoretical function of Eq. 1, using as free parameters $\Lambda_{\phi \mu}$ and an overall normalization factor. As result of the fit procedure, we determine a value for the form factor slope:

$$
\mathrm{b}_{\phi \eta}=(1.17 \pm 0.10) \mathrm{GeV}^{-2}
$$

with $\chi^{2} / \mathrm{NDF}=1.17$ and $\operatorname{Prob}\left(\chi^{2}\right) \approx 12.58 \%$. The value found for the normalization parameter is $(108.6 \pm 0.7) \times 10^{6}$ with a correlation of $39 \%$. In Figure 8 the fit result is shown for each invariant mass bin and compared with the data. The result is in agreement with the VDM model. The central value is smaller, although still consistent within the error, than the result obtained by the SND experiment [4].
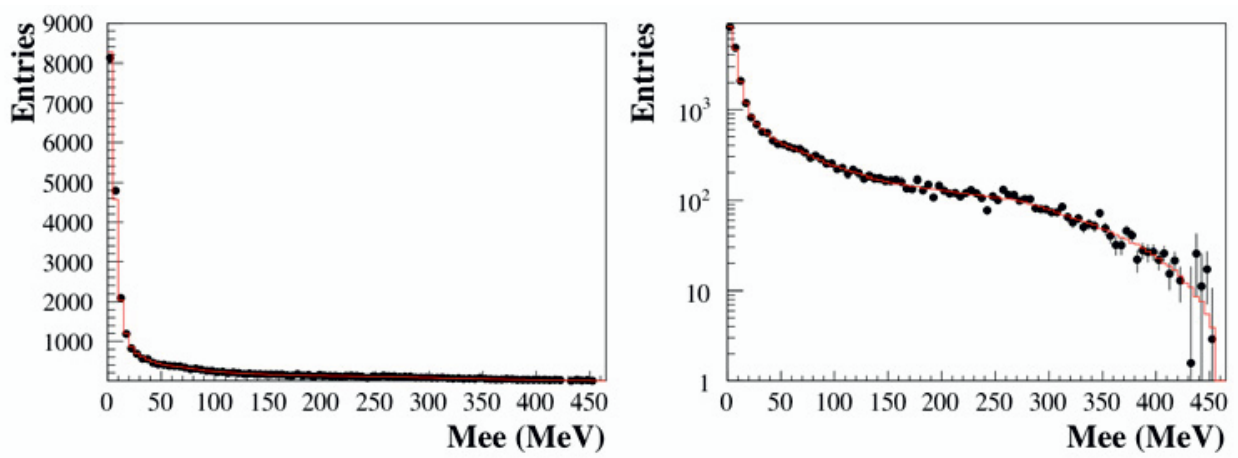

Figure 8. Fit to the $\mathrm{M}_{e e}$ spectrum for $\phi \rightarrow \eta e^{+} e^{-}$candidates, both in linear (left) e logarithmic (right) scale. 


\section{The $\phi \rightarrow \pi^{0} e^{+} e^{-}$analysis}

The total absence of experimental determination of the $F_{\phi \pi^{0} \gamma^{*}}$ transition form factor and the low accuracy in the knowledge of the branching ratio ( $~ 25 \%$ in the Particle Data Group world average), motivate the analysis of $\phi \rightarrow \pi^{0} e^{+} e^{-}$decay at KLOE. The signal events are selected requiring two tracks of opposite charge and two prompt neutral clusters coming from the IP. Simple kinematical cuts on the lepton energies $\left(E_{e^{+}, e^{-}}<460 \mathrm{MeV}\right.$ and $\left.470<E_{e^{+}}+E_{e^{-}}<750 \mathrm{MeV}\right)$, photon energies $\left(E_{\gamma_{1}, \gamma_{2}}>70 \mathrm{MeV}\right.$ and $\left.300<E_{\gamma_{1}}+E_{\gamma_{2}}<670 \mathrm{MeV}\right)$ and opening-angles of tracks and prompt clusters $\left(\theta_{e^{+} e^{-}}^{\text {open }}<145^{\circ}\right.$ and $\left.27^{\circ}<\theta_{\gamma_{1} \gamma_{2}}^{\text {open }}<57^{\circ}\right)$ allow to reject a big amount of dominant background from radiative Bhabha scattering processes (i.e. $e^{+} e^{-} \rightarrow e^{+} e^{-} \gamma \gamma$ ). The other main background contribution comes from the radiative decay $\phi \rightarrow \pi^{0} \gamma$, with the real photon converting to an $e^{+} e^{-}$pair on the beampipe and drift-chamber walls. This kind of events can be partially rejected tracking the leptons back to the conversion wall, and then cutting on the invariant-mass and distance between the tracks. The agreement between data and the sum of MC signal and background distribution is shown in Fig. 9 for several variables.
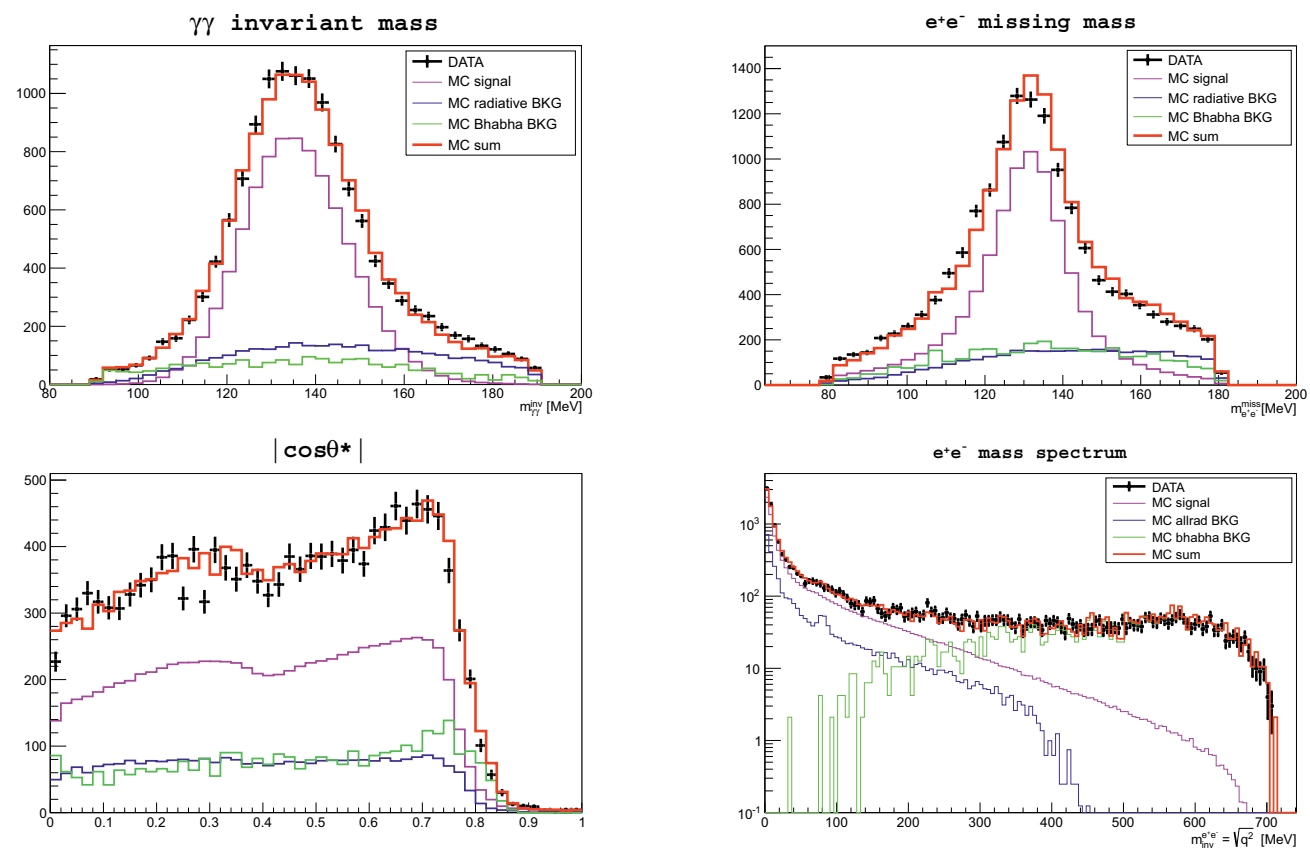

Figure 9. Comparison between data (black points) and MC distributions (red histogram is the MC sum) for the invariant mass of the two photons (top left), for the missing-mass to tracks (top right), for the angle between the momentum direction of $\phi$ and the $e^{+}$in the rest-frame of the lepton-pair (bottom left) and for the $e^{+} e^{-}$invariantmass spectrum (bottom right).

At the end of the analysis path, $\sim 14600$ events are selected, with a background contamination of about $\sim 35 \%$. The background subtraction, the extraction of the form factor and the BR are going to be finalized soon. 


\section{References}

[1] L.G. Landsberg, Phys. Rep. 128, 301 (1985);

[2] C. Tershlusen and S. Leupold, Phys. Lett. B 691, 191 (2010);

[3] S. Ivashyn., Prob.Atomic Sci.Technol. 2012N1, 179:182 (2012);

[4] M. N. Achasov et al. [SND collaboration], Phys. Lett. B 504, 275 (2001);

[5] R.R. Akhmetshin et al. [CMD-2 collaboration], Phys.Lett. B 503, 237 (2001);

[6] S. Giovannella, R. Versaci, KLOE Memo 350 (2008);

[7] F. Ambrosino et al. [KLOE collaboration], Eur. Phys. J c47, 589 (2006);

[8] S. Giovannela, S. Miscetti, KLOE note 177 (2002);

[9] Phys. Rev. D, 86 (2012);

[10] http://wwwasdoc.web.cern.ch/wwwasdoc/minuit/minuit.ps; 